\title{
Uncommon clinical presentations of large intestine injury-Report of two cases
}

\author{
Jacek Karoń ${ }^{1}$, Karolina Horbacka $^{1^{*}}$, Mariusz Stawiński $^{2}$, Mateusz Michalski ${ }^{2}$, Piotr Krokowicz $^{1}$ \\ ${ }^{1}$ Department of General and Colorectal Surgery, University of Medical Sciences, Poznań, Poland; \\ *Corresponding Author: karolinahorbacka@hotmail.com \\ ${ }^{2}$ Department of General, Laparoscopic and Endocrine Surgery, Poznań, Poland
}

Received 28 November 2013; revised 25 December 2013; accepted 20 January 2014

Copyright (C) 2014 Jacek Karon et al. This is an open access article distributed under the Creative Commons Attribution License, which permits unrestricted use, distribution, and reproduction in any medium, provided the original work is properly cited. In accordance of the Creative Commons Attribution License all Copyrights (C) 2014 are reserved for SCIRP and the owner of the intellectual property Jacek Karoń et al. All Copyright (C) 2014 are guarded by law and by SCIRP as a guardian.

\section{ABSTRACT}

We present an unusual clinical manifestation of a rectal rupture discussing two cases of female patients hospitalized and operated on urgently. The extraordinary presentations during physical examination, which eventually provided a solid indication for immediate operation included sigmoid colon prolapse in one case and evisceration of small intestine through the anus in the other. In both patients, Hartmann procedure was successfully performed resulting in relatively fast recovery despite an elderly age and hospital discharge on the $10^{\text {th }}$ postoperative day. Although sigmoid colon or ileal prolapse is not commonly observed in bizarre medical condition, they may be the first symptoms of rectal/rectosigmoid injuries as a result of underlying colon disorder such as diverticular disease, spontaneous rupture or self-injury.

\section{KEYWORDS}

\section{Rectal Tear; Transanal Small Bowel Evisceration; Hartmann Procedure}

\section{INTRODUCTION}

Obviously colon or ileal prolapse is a condition rarely observed and reported in daily surgical practice. However, they may be the first visible symptoms of rectosigmoid lacerations observed usually among the elderly patients, which are worth of notice in terms of the consequences of population ageing process and the challenges. It is created for medical care and surgery. Mortality associated with emergency abdominal surgery in the elderly is quite high but prompt diagnosis and adequate treat- ment strategy are essential for successful recovery.

\section{CASE REPORT 1}

A 71-year-old woman (G. J, history number: 31272/ 09/10C20023) was admitted to the Department of General and Colorectal Surgery University of Medical Sciences in Poznań on January 3, 2010 because of a large intestine prolapsing through her anus. On first sight the surgeon who admitted the patient at the Emergency Department had the impression that he deals with typical but significant rectal mucosal prolapse. After a thorough examination it appeared to be a part of a large bowel with mesentery and epiploic appendices (most probably — sigmoid colon), without any signs of mucosal prolapse (Figure 1(a)). Due to the patient's impaired mental status caused by a severe form of Parkinson's disease, obtaining information concerning a supposed mechanism explaining her condition was impossible.

On physical examination abdomen was soft, painless, without rebound tenderness, without distension, peristal-

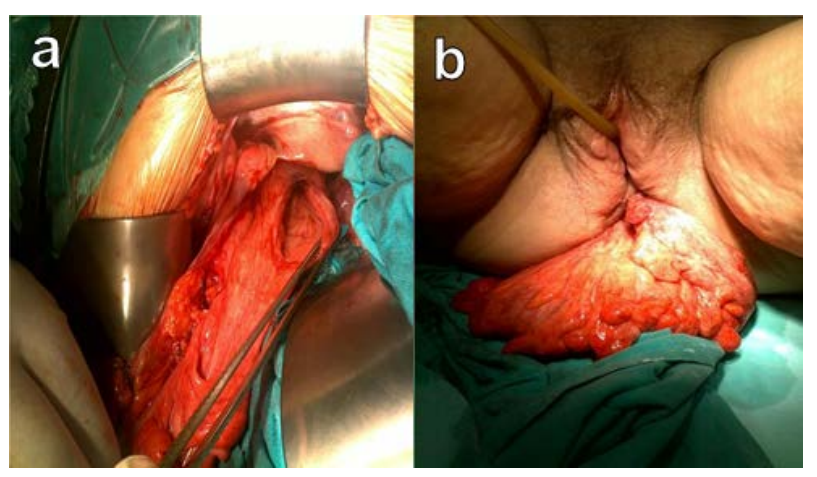

Figure 1. Sigmoid colon prolapsing through the anus (a); Intraoperative image at laparotomy demonstrating rectal injury (b). 
sis was not audible. The herniated sigmoid colon, with slight swelling and ischemia, without any accessory signs of mucosal rectal prolapse. Gynecological examination did not reveal any vaginal injuries. After inserting Foley catheter urine was clear. Temperature and vital signs were stable. Laboratory tests: hemoglobin-7.1 $\mathrm{mmol} / \mathrm{l}$, red blood cell count $-4.07 \times 10^{6} / \mu \mathrm{l}$, white blood cell count-18.7 $\times 10^{3} / \mu$ l, electrolytes, blood urea nitrogen, creatinine, glucose, amylase, bilirubin, aminotransferases within the recommended range. Coagulation parameters within normal limits. Ultrasound did not reveal any fluid in the peritoneal cavity.

Suspecting large bowel injury of unknown origin the decision for urgent operation was made. The abdominal cavity was opened via ventral midline incision. The loops of small intestine did not reveal any macroscopic changes, with normal peristalsis and no signs of inflammatory fluid or blood in the peritoneal cavity. Large bowel was impacted with hard, fecal stones.

A $5 \mathrm{~cm}$ long longitudinal laceration was found in the anterior rectal wall in the intraperitoneal part, just above the peritoneal fold. The loop of elongated sigmoid colon invaginated to the rectum through this rupture, eventually prolapsing through the anus (Figure 1(b)). Extruding intestine was repositioned through the rectal tear to the abdominal cavity and Hartmann procedure was performed: rectum was closed using linear stapler below the injury, elongated sigmoid colon, including the protruding part was excised and descending colon was brought out as a colostomy (Figure 2). One catheter was placed into the Douglas pouch. The operation finished with a profuse saline peritoneal lavage. Postoperative period was uneventful and the patient was eventually discharged home in a good condition on the $10^{\text {th }}$ day after the operation.

During hospitalization and psychological counseling we suspected that the most probable cause of rectal tear was self-injury. The patient, most of the day lying in bed, suffered from chronic constipation, which she treated not

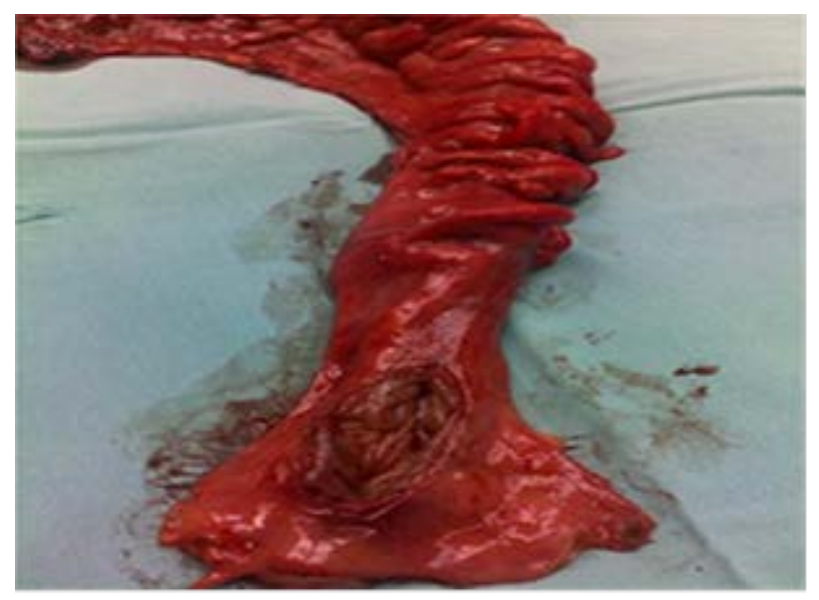

Figure 2. Resected part of the rectum and the sigmoid colon. only with laxatives, but also with mechanical removal of fecal stones using different devices (spoons, sticks, wooden tool handles).

\section{CASE REPORT 2}

A 79-year-old female patient (K.F., history number 28110/12/960/12/NCH) with a history of rectal prolapse presented to the Department of General, Laparoscopic and Endocrine Surgery in Poznań, on December 14, 2012 with a significant part of small bowel with mesentery protruding through her anus (Figures 3(a) and (b)). The patient's cognitive status was severely impaired as a consequence of dementia. According to the data obtained from the family members, the lady most of the day lying in bed had suffered from rectal and vaginal prolapse from 6 months. Till that time rectal prolapse had been successfully reduced with a help of the patient's caregiver.

During physical examination abdomen was soft, without tenderness or distention. The part of small intestine protruding out of the anus was swollen with signs of hyperemia. Gynecological examination revealed vaginal prolapse. After inserting Foley catheter urine was clear. The patient was afebrile with stable vital signs. Laboratory tests: hemoglobin $-7 \mathrm{mmol} / \mathrm{l}$, red blood cell count$4.00 \times 10^{6} / \mu \mathrm{l}$, white blood cell count-10.6 × $10^{3} / \mu \mathrm{l}$, C-reactive protein-10.5 mg/l, electrolytes, blood urea nitrogen, creatinine, glucose, amylase, bilirubin, aminotransferases within a normal range. Coagulation parameters within normal limits.

The patient was qualified for immediate operation. After entering abdominal cavity through midline incision no signs of fecal soilage, fluid or blood were present. Following a profuse irrigation of extruding small bowel

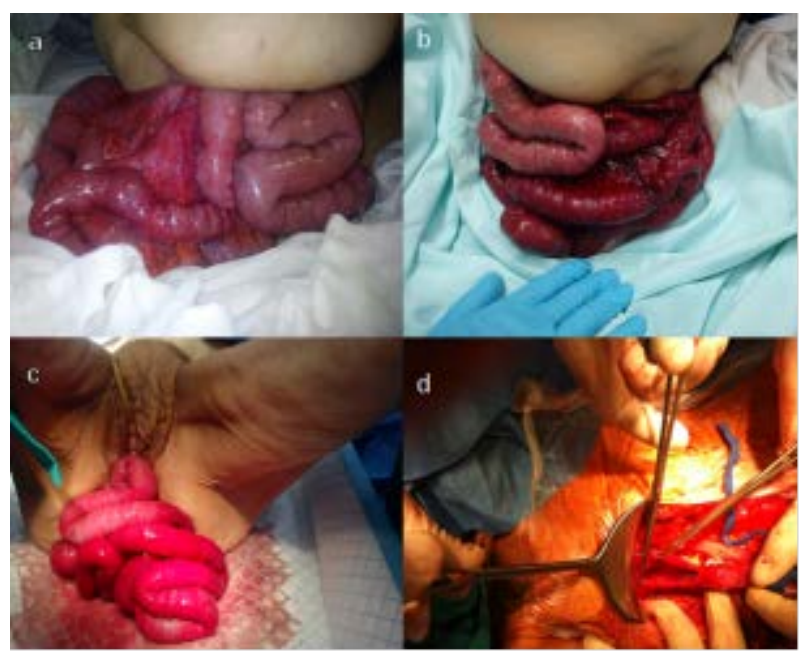

Figure 3. Transanal evisceration of small intestine (a); (b); (c) Intraoperative image at laparotomy demonstrating longitudinal tear in the anterior rectosigmoid wall (d). 
with saline solution it was diverted back to the abdominal cavity and after injection of a mesentery with lignocaine it resembled normal and viable suggesting a sufficient blood supply. Small intestine did not reveal any lesions but we found sigmoid colon diverticulosis complicated by the $3-4 \mathrm{~cm}$ long tear in the anterior side of rectosigmoid (Figure 3(d)), which formed the orfice for the small intestine to protrude through the rectal lumen directly out of the anus. The mechanism of the lesion could be potentially explained by diverticular perforation. Diverticular rectosigmoid colon was resected and Hartmann procedure was performed. The operation finished with a thorough irrigation of abdominal cavity, catheter was placed in the Douglas pouch. Postoperative course was complicated by myocardial infarction and the patient was observed for 3 days in the Intensive Care Unit. Fortunately, she was eventually discharged home on the $10^{\text {th }}$ day after the operation in a good condition, with healed abdominal wound and correct colostomy function.

\section{DISCUSSION}

Protrusion of small bowel loops or sigmoid colon through the anus is not commonly seen entity, however there has been quite a few such cases described in the literature since Benjamin Brodie first had reported a case of transanal ileal evisceration in 1827 [1]. The above mentioned condition is usually caused by rectal or distal sigmoid rupture as a consequence of trauma injury, underlying disease such as diverticulosis causing weakening of the intestinal wall or increase in abdominal pressure most common in the cases with coexisting rectal or uterine prolapse [2,3]. In the light of some reported series, about $70 \%$ cases of transanal ileal herniation were associated with rectal prolapse $[1,4]$. With regard to this theory, the episodes of rectal prolapse weaken intestinal wall, and moreover the increase in abdominal pressure pushes the viscera to create additional stress force resulting in perforation of large bowel. Considering this thesis in the second reported case, it had the patient not only with a history of rectal and vaginal prolapse but also a suffering of diverticular disease, which all may be interpreted as precipitating factors leading to rectal laceration with subsequent transanal small bowel evisceration.

One extraordinary aspect of those case reports is that despite the significant tear of the anterior rectal wall in its intraperitoneal part, no clinical or intraoperative signs of acute peritonitis were present. Most probably, the patients avoided this obvious consequence of intraperitoneal rectal rupture, because the proximal part of large bowel was completely impacted with fecal stones without any liquid content and thanks to the fact that the invagination of elongated sigmoid colon in the first case and small intestine in the other occurred pretty early.
Invaginated sigmoid colon in the first mentioned patient and ileal loops in the second in fact effectively occluded the rectal lesion, preventing the massive peritoneal contamination.

The management of rectal/rectosigmoid injuries complicated by the ominous evisceration of intestines requires reduction of an extruded bowel, followed by examining for viability with resection of a necrotic part. The lesion of large bowel can be either repaired with sutures or resected depending on patient's condition, size of perforation, blood supply, presence of peritoneal contamination or underlying intestinal disease $[5,6]$. Bringing out proximal ostomy is reasonable in case of any doubts concerning the suture tightness and strength. In both our patients, Hartmann procedure was performed resulting in relatively fast and successful recovery, which is crucial in terms of high mortality and postoperative complication rate after gastrointestinal operations observed among the elderly suffering from multiple concomitant disorders.

\section{REFERENCES}

[1] Wrobleski, D.E. and Dailey, T.H. (1979) Spontaneous rupture of the distal colon with evisceration of small intestine through the anus: Report of two cases and review of the literature. Diseases of the Colon and Rectum, 22, 569-572. http://dx.doi.org/10.1007/BF02587009

[2] Joon, J., Joon, S.P., Chang, G.B., Dong, S.Y., Seung, K.S., Yoon, H.L., et al. (2000) Rupture of the sigmoid colon with evisceration of the small bowel through the anus. Yonsei Medical Journal, 41, 289-292.

[3] Furuya, Y., Yasuhara, H., Naka, S., Yamazaki, K., Yanagie, H., Wakahara, T., et al. (2008) Intestinal evisceration through the anus caused by fragile rectal wall. International Journal of Colorectal Diseases, 23, 721-722. http://dx.doi.org/10.1007/s00384-008-0459-3

[4] Czerniak, A., Avigad, I., Vermesh, M., Rabau, M. and Wolfstein, I. (1983) Spontaneus rupture of rectosigmoid colon with small bowel evisceration through the anus. Report of a case and review of the literature. Diseases of the Colon and Rectum, 26, 821-822. http://dx.doi.org/10.1007/BF02554760

[5] Shoab, S.S., Saravanan, B., Neminathan, S. and Garsaa, T. (2007) Thiersch repair of a spontaneous rupture of rectal prolapse with evisceration of small bowel through anusa case report. Annals of the Royal College of Surgeons of England, 89, W6-W8.

http://dx.doi.org/10.1308/147870807X160362

[6] Papapolychroniadis, C., Kaimakis, D., Giannoulis, K., Parlapani, A., Karpini, E., Georgiou, M., et al. (2004) Spontaneous rupture of the rectosigmoid with small bowel evisceration through the anus. Techniques in Coloproctology, 8, S126-S128. http://dx.doi.org/10.1007/s10151-004-0132-y 